\title{
Avaliação de RNAs Durante Treinamento Supervisionado Para Classificação de Adolescentes com Autismo
}

\author{
Rhyan Ximenes de Brito ${ }^{1}$, Carlos Alexandre Rolim Fernandes ${ }^{2}$, \\ Janaide Nogueira de Sousa Ximenes ${ }^{3}$ \\ ${ }^{1}$ Instituto Federal de Educação, Ciência e Tecnologia do Ceará (IFCE) \\ ${ }^{2}$ Universidade Federal do Ceará (UFC) \\ ${ }^{3}$ Faculdade IEducare (FIED) \\ \{rxbrito, nogueirajanaide\}@gmail.com, alexandre_ufc@yahoo.com.br
}

\begin{abstract}
Several techniques of Artificial Intelligence have been widely used in the solution of several problems in the health area. In this perspective, in this work, a study was carried out through the implementation and analysis of the neural networks ELM (Extreme Learning Machine) and MLP (Multilayer Perceptron), comparing the accuracy resulting from training with data from adolescents with or without ASD (Autistic Spectrum Disorder). The methodology was based on a public database of 99 samples and the $k$-fold cross-validation technique with and without zscore normalization. Regarding the results, the MLP network without zscore had the best average reaching $89.70 \%$ of correct answers, however the ELM network without zscore had the worst average of correct answers with $86.52 \%$.
\end{abstract}

Resumo. Diversas técnicas de Inteligência Artificial tem sido amplamente utilizadas na solução de diversos problemas na área de saúde. Neste trabalho foi realizado um estudo através da implementação e análise das redes neurais ELM (Extreme Learning Machine) e MLP (Multilayer Perceptron), comparando as acurácias resultantes de treinamentos com dados de adolescentes com ou sem o TEA (Transtorno do Especto Autista). A metodologia foi baseada em um banco de dados público de 99 amostras e na técnica de validação cruzada $k$-fold com e sem normalização zscore. Com relação aos resultados a rede MLP sem zscore obteve a melhor média atingindo $89,70 \%$ de acertos, contudo a rede ELM sem zscore teve a pior media de acertos com $86,52 \%$.

\section{Introdução}

O TEA (Transtorno do Especto Autista) é um transtorno de início precoce que afeta predominantemente três habilidades: interação social, comunicação ou linguagem e comportamento [de Moura et al. 2018]. Várias teorias foram criadas na tentativa de explicar as características presentes em pessoas com autismo, contudo a etiologia permanece desconhecida [de Moura et al. 2018].

De acordo com [Thabtah 2017], o TEA é um dos principais distúrbios psiquiátricos que limita o uso de habilidades linguísticas, comunicativas, cognitivas, bem como sociais e habilidades. O TEA tem sido estudado na área comportamental, incluindo 
métodos inteligentes baseados em aprendizado de máquina para acelerar o tempo de triagem ou para melhorar a sensibilidade, especificidade ou precisão do processo de diagnóstico.

Este trabalho teve como objetivo realizar um estudo através da implementação e análise das redes neurais ELM (Extreme Learning Machine) e MLP (Multilayer Perceptron), comparando as acurácias resultantes de treinamentos com dados de adolescentes com ou sem o TEA, auxiliando na classificação desse grupo de pessoas.

A metodologia aplicada nesse trabalho foi desenvolvida com base em um grupo de indivíduos do banco de dados público "Autistic Spectrum Disorder Screening Data for Adolescent" obtido através do link, https://archive.ics.uci.edu/ml/machine-learningdatabases/00420/.

Este trabalho está organizado da seguinte forma: a Seção 2 apresentará os trabalhos relacionados, a Seção 3 abordará o TEA, a Seção 4 ressaltará as redes neurais artificiais (MLP e ELM), a Seção 5 informará a metodologia utilizada, a Seção 6 mostrará os resultados e discussões e por fim, conclusões e trabalhos futuros serão tratados na Seção 7.

\section{Trabalhos Relacionados}

Esta seção tem como finalidade apresentar algumas abordagens relacionadas à utilização de técnicas de Inteligência Artificial que buscam por respostas relacionadas a diferentes problemas pesquisados na área de saúde.

[Linstead et al. 2015] aplicou redes neurais artificiais em uma base de dados com 726 amostras de crianças com transtorno do espectro autista para prever o domínio dos resultados da aprendizagem em resposta à terapia comportamental. $\mathrm{O}$ resultados mostram que as redes neurais superam substancialmente os modelos de regressão linear relatados no domínio da pesquisa em autismo.

[da Silva et al. 2018] propôs levar o conhecimento e veicular informação a respeito do TEA e IA, através da criação de um jogo com base nas principais metodologias de análise comportamental, ABA (Applied Behavior Analysis) e TEACCH (Treatment and Education of Autistic and Related Communication Handicapped Children). Dessa forma, criaram um jogo customizável capaz de interagir com o neuroatipico de forma inteligente e desestigmatizada, apresentando de forma divertida a interação social, cognitiva, pessoal e didática.

[Thabtah 2017] trouxe uma discussão sobre o emprego do aprendizado de máquina na classificação do autismo discutindo os prós e contras, destacando problemas associados as atuais ferramentas de triagem, com foco na confiabilidade usando o DSM-4 (Diagnostic and Statistical Manual of Mental Disorders-4) em vez da nova classificação do DSM-5.

[Thabtah 2019] analisou criticamente estudos investigativos recentes sobre o TEA, não apenas articulando questões como habilidades linguísticas de comunicação, cognitivas e sociais, mas também recomendando caminhos a seguir que aprimoram o uso do aprendizado de máquina no TEA com relação à conceitualização e implementação de dados. 
Faz-se importante salientar que durante a elaboração deste trabalho foi observada a carência de estudos utilizando classificadores com foco no TEA em adolescentes.

\section{O Transtorno do Espectro Autista}

O autismo foi determinado por Kanner em 1943, porém só foi reconhecido oficialmente por volta de 1994 na DSM-4 (Manual de Diagnóstico e Estatístico de Transtornos Mentais $4^{\mathrm{a}}$ edição) como autismo e, a partir do DSM-5 (Manual de Diagnóstico e Estatístico de Transtornos Mentais $5^{\mathrm{a}}$ edição), passou a ser conhecido como TEA.

Sabe-se que o TEA afeta áreas do neurodesenvolvimento responsáveis pela interação social, comunicação e comportamento, necessita de intervenções específicas e precoces para potencializar o desenvolvimento infantil, diminuir a possibilidade de cronificação e ampliar propostas terapêuticas [Zanon et al. 2014].

O diagnóstico do TEA é prevalente no contexto infanto-juvenil, afetando diversos aspectos da cognição apresentando manifestações comportamentais significativas [Fernandes et al. 2018]. Não existe uma cura para o TEA, para se atenuar os efeitos podem ser feitas intervenções com base em modelos comportamentais [de Brito et al. 2019].

Vale ressaltar que os diagnósticos convencionais para o TEA exigem que os médicos conduzam uma avaliação clínica da idade de desenvolvimento do paciente com base em vários domínios como por exemplo, excessos de comportamento, comunicação, autocuidado e habilidades sociais [Thabtah et al. 2018].

Atualmente tem-se como referência o DSM-5, que aborda uma nova classificação abrangendo quatro condições assim assinaladas: déficit em comunicação; em interação social; em padrão de comportamento; e atividades e interesses restritivos e repetitivos [Freire Monteiro et al. 2018].

Dessa forma, entende-se haver um consenso científico de que esses quatro distúrbios corresponderiam a uma mesma condição, para a qual há diferentes níveis de severidade dos sintomas, seguindo as ponderações da APA (Associação Americana de Psiquiatria) apresentadas no manual de diagnóstico (DSM-5) [Freire Monteiro et al. 2018].

\section{Redes Neurais Artificiais}

As RNA (Redes Neurais Artificiais) são sistemas computacionais adaptativos inspirados no processamento de informações realizado por neurônios biológicos tal como aqueles existentes em organismos inteligentes [Neto et al. 2017].

\subsection{Extreme Learning Machine (ELM)}

A ELM tem recebido bastante atenção da comunidade científica nos últmos anos. Esta rede utiliza o conceito de projeção aleatória, onde os pesos da camada oculta são escolhidos aleatoriamente e não há treinamento para ajustá-los [Silvestre 2014].

O treinamento consiste em ajustar de forma analítica os pesos da camada de saída, sendo necessário projetar os dados em um espaço de mais alta dimensão. Na camada oculta o número de neurônios é consideravelmente maior do que o de uma SLFN (SingleLayered Feedforward Neural Network), rede que apresenta apenas uma camada oculta, treinada com um algoritmo como o de retropropagação de erros [Silvestre 2014]. 
As ELMs apresentam algumas características importantes, uma delas é a rapidez do treinamento por não envolver um processo iterativo como no caso das MLPs, o tempo necessário para o treinamento costuma ser significativamente menor que aquele despendido por outras técnicas [Souza 2016].

Abaixo a Figura 1 apresenta a estrutura básica de uma RNA do tipo ELM, sua arquitetura é semelhante a estrutura da rede MLP.

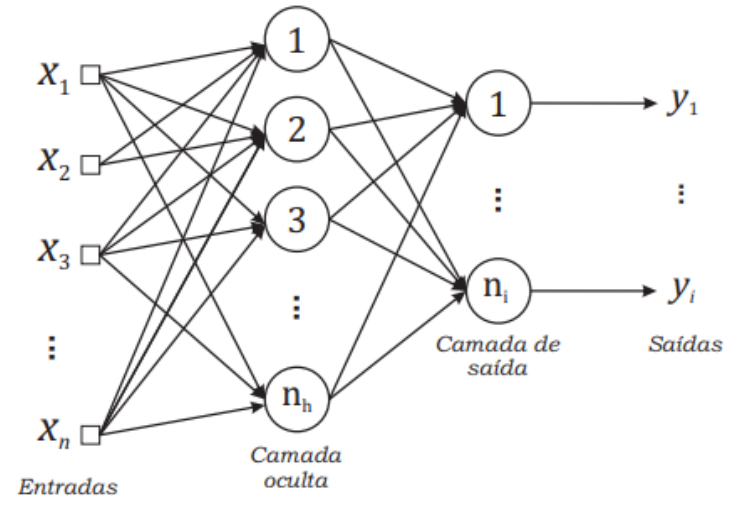

Figura 1. Extreme Learning Machine (ELM)

\subsection{Multilayer Perceptron (MLP)}

De acordo com [Bonifácio 2010], uma rede MLP consiste de uma camada de entrada, uma ou mais camadas ocultas e uma camada de saída. É do tipo feedforward, ou seja, nenhuma saída de um neurônio de uma camada k será sinal de entrada para um neurônio de uma camada menor ou igual a $\mathrm{k}$, e é completamente conectada, de forma que cada neurônio fornece sua saída para cada unidade da camada seguinte.

As MLPs são normalmente aplicadas em problemas de classificação, de aproximação (ou análise de regressão) incluindo previsão e modelagem de séries temporais em áreas como: controle, diagnóstico e data mining [Ferreira et al. 2016]. A Figura 2 apresenta a estrutura básica de uma rede neural artificial MLP.

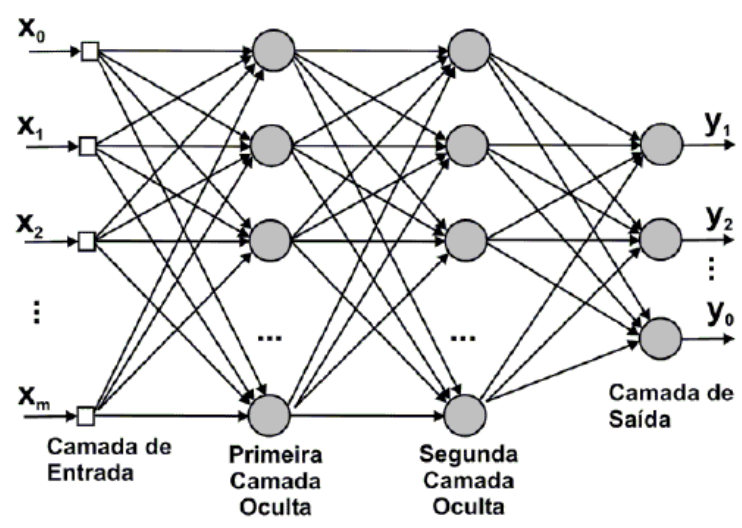

Figura 2. Multilayer Perceptron (MLP) 


\section{Metodologia}

A abordagem proposta nesse estudo está fundamentada na classificação de dados através da utilização das RNAs ELM e MLP. A metodologia desse trabalho foi desenvolvida em etapas: (1) balanceamento da base de dados utilizada de forma a garantir a proporcionalidade do número de amostras de cada classe; (2) treinamento e; (3) teste com a base de dados, nessa ordem.

\subsection{Base de Dados Utilizada}

A implementação dos algoritmos foi realizada a partir de um banco de dados público intitulado "Autistic Spectrum Disorder Screening Data for Adolescent "disponível através do link: https://archive.ics.uci.edu/ml/machine-learning-databases/00420/, composto por 2 classes (indivíduo com ou sem TEA), 99 amostras e 20 atributos, criada e implementada em sua totalidade por [Thabtah 2019], com dados de adolescentes de várias partes do mundo, alimentada através do aplicativo ASD tests disponível na google play e app store.

Salienta-se que na base de dados existem 62 amostras de adolescentes com TEA $(63,26 \%)$ e 37 amostras de adolescentes sem o transtorno (36,74\%). Ressalta-se que foi utilizada a validação cruzada com $k$-fold com $\mathrm{k}=10$, com dados separados em 10 conjuntos balanceados.

\subsection{Treinamento e Teste com a Base de Dados}

Para o treinamento e teste das redes foram considerados os seguintes cenários: (i) dados normalizados utilizando o zscore; (ii) e sem usar o zscore. Vale destacar que a técnica cross validation $k$-fold é um método de teste existente no aprendizado de máquina para examinar os modelos preditivos e avaliar sua eficácia [Thabtah 2019].

Ressalta-se ainda que os dados da base foram embaralhados aleatóriamente de forma a criar instâncias para cada classe em cada partição. Esse processo é chamado estratificação, portanto, o método de teste é conhecido como validação cruzada estratificada [Thabtah 2019].

Para a escolha da configuração da rede neural ELM testou-se várias configurações até encontrar a que obtivesse a melhor performance de acertos, constantando-se que com 1 camada de entrada com 19 nerônios, 1 camada oculta com 10 e uma de saída com 2 neurônios.

Já para a escolha da configuração da rede MLP embasou-se no desempenho entre várias configurações testadas. Assim para o treino e teste com arquitetura MLP foram utilizados 19 neurônios na camada de entrada, 2 camadas ocultas com 10 neurônios cada e uma camada de saída com 2 neurônios, para o treinamento utilizou-se 20.000 epochs.

\section{Resultados e Discussões}

Essa seção trará discussões a cerca dos resultados adquiridos conforme é evidenciado nas tabelas e matrizes de confusão abaixo, desenvolvidas com base nas redes MLP e ELM.

As matrizes de confusão apresentadas possuem a seguinte estrutura: as linhas correspondem à classe prevista e as colunas correspondem a classe verdadeira. As células diagonais correspondem as classificações corretas e as células fora da diagonal correspondem as classificações incorretas. O número de amostras e a porcentagem do número 
total de amostras são mostrados em cada célula. A coluna da extrema direita da matriz e a linha na parte inferior mostram as porcentagens que pertencem a cada classe que foram classificadas correta e incorretamente.

A Figura 3 mostra a matriz de confusão da rede MLP sem zscore com taxa média de acertos de $89,70 \%$, obitida entre todos os folds dos testes realizados enquanto que com zscore foi de $87,52 \%$ de acerto.

\begin{tabular}{|c|c|c|c|}
\hline & 1 & 2 & \\
\hline & 29 & 8 & $78,38 \%$ \\
\hline & $29,67 \%$ & $8,08 \%$ & $21,62 \%$ \\
\hline & 4 & 58 & $93,55 \%$ \\
\hline & $2,22 \%$ & $60,03 \%$ & $6,45 \%$ \\
\hline & $87,88 \%$ & $87,88 \%$ & $89,70 \%$ \\
\hline & $12,12 \%$ & $12,12 \%$ & $10,30 \%$ \\
\hline
\end{tabular}

\section{Figura 3. Matriz de Confusão MLP sem Zscore}

A Figura 4 evidencia a taxa média entre os folds dos testes realizados na rede ELM com zscore atingindo $89,51 \%$ de acerto. Vale ressaltar que sem normalização zscore a taxa foi de $86,52 \%$ de acerto.

\begin{tabular}{|c|c|c|c|}
\hline & 1 & \multicolumn{2}{|l|}{2} \\
\hline & 31 & 8 & $79,48 \%$ \\
\hline & $32,12 \%$ & $7,08 \%$ & $20,52 \%$ \\
\hline & 4 & 56 & $93,33 \%$ \\
\hline & $3,41 \%$ & $57,39 \%$ & $6,67 \%$ \\
\hline & $88,58 \%$ & $87,50 \%$ & $89,51 \%$ \\
\hline & $11,42 \%$ & $12,50 \%$ & $10,49 \%$ \\
\hline
\end{tabular}

Figura 4. Matriz de Confusão ELM com Zscore

Ficando evidente que entre todos os testes realizados as redes MLP sem zscore e a ELM com zscore obtiveram respectivamente como taxa média de acerto $89,70 \%$ e $89,51 \%$, configurando-se assim como os melhores resultados entre todos os testes realizados.

Logo em seguida tem-se as Tabela 1 e Tabela 2 com as taxas de acerto para cada um dos folds nos testes realizados com a base de dados na arquitetura MLP sem a normalização zscore e ELM com normalização zscore.

Assim, à Tabela 1 com resultados da MLP sem normalização evidencia-se que os folds 3,4 e 10 forneceram os melhores resultados com 100\% de acerto, enquanto que o fold 9 forneceu o pior resultado com $66,70 \%$ de acerto.

Já a Tabela 2 contém os resultados com a arquitetura ELM com normalização zscore. Pode-se notar que os folds 1, 3, 5, 7 e 9 obtiveram os melhores resultados com $100 \%$ de acertos, enquanto que o pior resultado foi do fold 6 com $63,60 \%$ de acerto.

Como pode ser constatado, a rede MLP sem normalização zscore obteve a melhor média entre as demais, atingindo $89,70 \%$ de acerto, sendo que a pior média de acertos foi da rede neural ELM sem normalização zscore, atingindo 86,52\% de acerto. Lembrando que a ELM com normalização zscore, atingiu 89,51\% de acerto, conforme Tabela 2. 
Tabela 1. Resultados dos Testes (MLP sem Dados Nornalizados)

\begin{tabular}{cccc}
\hline$K$-fold & Taxa de Acerto Geral (\%) & Taxa de Erro Geral (\%) & Situação \\
\hline $1^{\circ}$ & $90,90 \%$ & $9,10 \%$ & - \\
$2^{\circ}$ & $90,90 \%$ & $9,10 \%$ & - \\
$3^{\circ}$ & $100,00 \%$ & $0,00 \%$ & Melhor Caso \\
$4^{\mathrm{o}}$ & $100,00 \%$ & $0,00 \%$ & Melhor Caso \\
$5^{\circ}$ & $90,90 \%$ & $9,10 \%$ & - \\
$6^{\circ}$ & $90,90 \%$ & $9,10 \%$ & - \\
$7^{\circ}$ & $88,90 \%$ & $11,10 \%$ & - \\
$8^{\circ}$ & $77,80 \%$ & $22,20 \%$ & - \\
$9^{\mathrm{o}}$ & $66,70 \%$ & $33,30 \%$ & Pior Caso \\
$10^{\circ}$ & $100,00 \%$ & $0,00 \%$ & Melhor Caso \\
Taxa média & $89,70 \%$ & $10,30 \%$ & Médio Caso \\
\hline
\end{tabular}

Tabela 2. Resultados dos Testes (ELM com Dados Nornalizados)

\begin{tabular}{cccc}
\hline$K$-fold & Taxa de Acerto Geral (\%) & Taxa de Erro Geral (\%) & Situação \\
\hline $1^{\mathrm{o}}$ & $100,00 \%$ & $0,00 \%$ & Melhor Caso \\
$2^{\mathrm{o}}$ & $84,20 \%$ & $15,80 \%$ & - \\
$3^{\mathrm{o}}$ & $100,00 \%$ & $0,00 \%$ & Melhor Caso \\
$4^{\mathrm{o}}$ & $84,20 \%$ & $15,80 \%$ & - \\
$5^{\mathrm{o}}$ & $100,00 \%$ & $0,00 \%$ & Melhor Caso \\
$6^{\mathrm{o}}$ & $63,60 \%$ & $36,40 \%$ & Pior Caso \\
$7^{\mathrm{o}}$ & $100,00 \%$ & $0,00 \%$ & Melhor Caso \\
$8^{\mathrm{o}}$ & $84,20 \%$ & $15,80 \%$ & - \\
$9^{\mathrm{o}}$ & $100,00 \%$ & $0,00 \%$ & Melhor Caso \\
$10^{\circ}$ & $78,90 \%$ & $21,10 \%$ & - \\
Taxa média & $89,51 \%$ & $10,49 \%$ & Médio Caso \\
\hline
\end{tabular}

\section{Conclusões de Trabalhos Futuros}

O artigo relatou a utilização de redes neurais artificiais como ferramentas de aprendizagem de máquina supervisionada abordando o autismo na adolescência como um problema de classificação de dados com base em um banco de dados composto por 99 amostras de adolescentes (62 autistas e 37 não autistas).

Assim constatou-se que a rede MLP sem normalização obteve a melhor média atingindo $89,70 \%$ de acertos e a ELM sem normalização o pior resultado com taxa média de $86,52 \%$ de acertos.

Sugere-se como trabalho futuro a implementação de outros classificadores como o SVM (Support Vector Machines) ou mesmo o Naive Bayes, com estratégias como leaveone-out ou hold- out comparando-as e analisando os resultados.

\section{Referências}

Bonifácio, F. N. (2010). Comparação entre as redes neurais artificiais MLP, RBF e LVQ na classificação de dados. Paraná: Universidade Estadual do Oeste do Paraná. 
da Silva, R. O., dos Santos, E. B., de Souza, E. M., and Nunes, W. B. (2018). Autismo: Interfaces da ti quebrando paradigmas. TECNOLOGIAS EM PROJEÇÃO, 9(1):01-08.

de Brito, R. X., Fernandes, C. R., and Amora, M. A. B. (2019). Análise de desempenho com redes neurais artificiais, arquiteturas MLP e RBF para um problema de classificação de crianças com autismo. iSys - Revista Brasileira de Sistemas de Informação, 12(1).

de Moura, P. J., Sato, F., and Mercadante, M. T. (2018). Bases neurobiológicas do autismo: enfoque no domínio da sociabilidade. Cadernos de Pós-Graduação em Distúrbios do Desenvolvimento, 5(1).

Fernandes, C. S., Fichman, H. C., and de Souza Barros, P. (2018). Evidências de diagnóstico diferencial entre transtorno do espectro autista (TEA) e transtorno do desenvolvimento intelectual (TDI): análise de casos. Neuropsicologia Latinoamericana, $10(2)$.

Ferreira, A., Ferreira, R. P., da Silva, A. M., Ferreira, A., and Sassi, R. J. (2016). Um estudo sobre previsão da demanda de encomendas utilizando uma rede neural artificial. Blucher Marine Engineering Proceedings, 2(1):353-364.

Freire Monteiro, A., de Almeida Pimenta, R., Pereira, S. M., and Roesler, H. (2018). Considerações sobre critérios diagnósticos de transtorno do espectro autista, e suas implicações no campo científico. DO CORPO: ciências e artes, 7(1).

Linstead, E., German, R., Dixon, D., Granpeesheh, D., Novack, M., and Powell, A. (2015). An application of neural networks to predicting mastery of learning outcomes in the treatment of autism spectrum disorder. In 2015 IEEE 14th International Conference on Machine Learning and Applications (ICMLA), pages 414-418.

Neto, A. B., Bonini, C. d. S. B., Bisi, B. S., dos Reis, A. R., and Coletta, L. F. S. (2017). Rede neural artificial para classificação e análise de solos degradados. IEEE Latin America Transactions, 15(3):503-509.

Silvestre, L. J. (2014). Regularização de extreme learning machines: uma abordagem com matrizes de afinidade.

Souza, L. L. d. (2016). Análise da pressão plantar da marcha de autistas por dinâmica simbólica otimizada por algoritmo genético.

Thabtah, F. (2017). Autism spectrum disorder screening: machine learning adaptation and DSM-5 fulfillment. In Proceedings of the 1st International Conference on Medical and health Informatics 2017, pages 1-6.

Thabtah, F. (2019). Machine learning in autistic spectrum disorder behavioral research: A review and ways forward. Informatics for Health and Social Care, 44(3):278-297.

Thabtah, F., Kamalov, F., and Rajab, K. (2018). A new computational intelligence approach to detect autistic features for autism screening. International journal of medical informatics, 117:112-124.

Zanon, R. B., Backes, B., and Bosa, C. A. (2014). Identificação dos primeiros sintomas do autismo pelos pais. Psicologia: Teoria e Pesquisa, 30(1):25-33. 